\section{Effect of handpiece light and material used in pulp chamber on dentin removal during root canal retreatment}

\author{
Marcio Alex Barros Gomes (D1, Igor Firmino Pereira Leão (D)', Laís Rani \\ Sales Oliveira (D1, Renata Afonso da Silva Pereira [1', Priscilla Barbosa \\ Ferreira Soares (102, Carlos José Soares ${ }^{1}$ (I) .
}

\begin{abstract}
This study evaluated the effect of the use of glass ionomer cement (GIC) and flowable bulk-fill resin composite (BFRC) for filling pulp chambers and the type of high-speed handpiece light used on dentin removal during access preparation for endodontic retreatment in molar teeth. Twenty maxillary molars were treated endodontically. BFRC (Opus Bulk Fill Flow APS, FGM) was used to fill the pulp chamber and replace coronal dentin $(n=10)$. In the remaining teeth, the pulp chamber was filled with GIC (Maxion R, FGM). Conventional resin composite (Opallis, FGM) was used to restore the enamel layer in all teeth. The samples in each group were divided into two subgroups, and the root canals were reaccessed using a handpiece with white or ultraviolet light. The teeth were scanned using micro-CT before and after root canal reaccess. The dentin volume removed was calculated and analyzed using 2 -way analysis of variance and Tukey's test $(\alpha=0.05)$. The crown and pulp chamber locations with dentin removal are described using frequency distribution. During the access, fewer pulp chamber walls were affected and a lower volume of dentin was removed from the pulpal floor in the group restored with GIC than in the group restored with BFRC. No effect was observed on the coronal dentin walls with respect to the filling protocols and type of light used. For dentin removal from the pulp chamber, handpieces with white light performed better than those with ultraviolet light, irrespective of the filling protocol used. The use of GIC to fill the pulp chamber and use of white handpiece light reduced dentin removal from the pulpal floor and resulted in fewer affected dentin walls.
\end{abstract}

${ }^{1}$ Department of Operative Dentistry and Dental Materials, School of Dentistry, Federal University of Uberlândia, Uberlândia, Minas Gerais, Brazil.

2Department of Periodontology and Implantology, School of Dentistry, Federal University of Uberlândia, Uberlândia, Minas Gerais, Brazil.
Correspondence: Carlos José Soares, Department of Operative Dentistry and Dental Materials, Federal University of Uberlândia, Av Pará 1720, Bloco 2B, Campus Umuarama, Uberlândia, MG 38400-902, Brazil. E-mail address: carlosjsoares@ufu.br Phone: +55 34999713472

\title{
Introduction
}

Several materials, such as conventional or bulk-fill resin composites (BFRC) with or without glass ionomer cement (GIC), have been used to restore posterior teeth after endodontic treatment (1), (2). The use of GIC for filling the pulp chamber has been implemented to reduce shrinkage stress (2). Definitive restoration is important for maintaining the quality of the endodontic treatment (3). When endodontic treatment fails, a new root canal access must be prepared to allow effective cleaning, shaping, and obturation of the root canal (4). The inability to distinguish between the tooth structure and restorative material during retreatment can result in damage to intact dentin on the lateral walls or at the pulp chamber floor because of the similarity between the resin composite and tooth structure (5). In extreme situations, accidental perforation of the pulp chamber floor can occur, which is a severe complication of endodontic and restorative procedures $(6,7)$, and is considered one of the major causes of failure of endodontic treatment (6).

The color and stiffness of the restorative material used in the pulp chamber can influence the identification of this material, preventing accidental dentin removal. The use of restorative materials such as GIC, which presents lower mechanical resistance (8) and higher color differentiation from the dentin when compared to resin composite (9), can facilitate the identification of the limit between the restorative material and dentin. A flowable resin composite that is easy to inject inside the pulp chamber is normally more translucent than GIC (10), and mimics the adjacent tooth structure; consequently, the identification of the limit might be difficult. A conventional high-speed handpiece with a diamond bur is used for the removal of restorative materials in coronal cavities and inside the pulp chamber $(5,11,12)$. Some restorative materials exhibit higher fluorescence, which makes them more visible when exposed 
to ultraviolet light (13). High-speed handpieces with ultraviolet LED headlamp systems have been propagated to highlight the fluorescence of these restorative materials, creating a contrast to that of the dental structure $(13,14)$. On the other hand, the high intensity of white light presented in handpieces can also improve visualization in deep areas of the pulp chamber $(5,12)$. Misplaced teeth, misidentification of the root canal, or an extra-coronal restoration are factors that may make root canal access difficult and predispose to root perforation (11). Illumination from the handpiece may facilitate the selective removal of composites and reduce the damage to intact dentin compared to that from conventional high-speed handpieces. To the best of our knowledge, no study has tested the efficiency of using GIC or a flowable resin composite layer for filling pulp chambers and that of using white or ultraviolet LED high-speed handpieces to prevent iatrogenic removal of intact dentin during endodontic access cavity preparation for retreatment.

Therefore, the aim of this study was to evaluate the influence of the type of restorative material used for filling endodontically treated molars and the type of light emitted by the headpiece during access preparation for retreatment of root canals in maxillary first molars on the removal of intact dentin and the location of dentin removal. The null hypothesis was that the restorative material used for filling the pulp chamber and the type of high-speed handpiece lighting would not influence dentin removal in endodontic retreatment access preparation in molar teeth.

\section{Materials and methods}

\section{Teeth selection and access}

This study was approved by the Ethics Committee (protocol \#2.570.199). The sample size was based on previous micro-CT studies (15). Twenty human maxillary first molar teeth extracted due to periodontal disease, and without any previous restoration or carious lesions, were selected. The inclusion criteria for molar teeth selection were coronal size and shape, and the volume and shape of the pulp chamber. Crown dimensions were measured using a digital caliper (Mitutoyo, Tokyo, Japan). The shape and area of the pulp chamber were checked on digital radiographs obtained using a photostimulable phosphor plate (VistaScan \#2; Durr Dental, Bietigheim Bissingen, Germany) and an X-ray machine (Timex 70 E, Gnatus, Ribeirão Preto, São Paulo, Brazil) positioned $20 \mathrm{~cm}$ from the specimen with an exposure time of $0.10 \mathrm{~s}$, operating at $70 \mathrm{kV}$ and $7.0 \mathrm{~mA}$. The dimensions were obtained using the DBSWIN software (VistaScan). Teeth with more than $10 \%$ variation in mean values were excluded. The teeth were embedded in a polystyrene resin (16). An endodontic access cavity was prepared using a round diamond bur (\#1014, KG Sorensen, Cotia, SP, Brazil) in a high-speed handpiece (Kavo, Joinville, SC, Brazil) under refrigeration to access the pulp chamber, and an Endo-Z drill (KG Sorensen, Cotia, SP, Brazil) was used to ensure a standardized cavity contour, allowing the location of the canal orifices. All procedures on all specimens were performed by the same experienced professional.

\section{Canal instrumentation and obturation}

The teeth were instrumented $1.0 \mathrm{~mm}$ short of the apical foramen. A Prodesign S system (Easy Equipamentos, Belo Horizonte, MG, Brazil) was used for irrigation with 2.5\% NaOCl (ASFER, São Caetano do Sul, SP, Brazil) using a 5-mL syringe (Ultradent, South Jordan, Utah, USA) and NaviTip (Ultradent, South Jordan, Utah, USA). AH Plus endodontic cement (Dentsply, Petrópolis, RJ, Brazil) and the lateral condensation technique were used for obturation, and the apical portion of the gutta-percha cone was kept $1.0 \mathrm{~mm}$ from the radiographic apex. Heated instruments (GP heater; Dentsply Maillefer) were used to remove the excess gutta-percha at the same level for all samples, that is, $0.5 \mathrm{~mm}$ below the pulp chamber floor, corresponding to the entrances of the root canals (Figure 1A-1B). The specimens were stabilized in a table device that standardized the position, orientation, and operator visualization of each specimen during the entire experiment. Only one operator, an endodontic specialist positioned ergonomically, prepared all root canal access cavities and performed all root canal treatments. A dental mirror and a sharp ended explorer (Golgran Millennium, São Caetano do Sul, SP, Brazil) were used for all procedures. The operator condition was standardized using appropriated conventional direct dental office lighting under a $3.5 \times$ magnification glass.

\section{Restorative procedures}

The endodontically treated teeth were randomly sorted into two groups $(n=10)$ using www.random.org. In group GIC (Figure 1A), a 1.5-2.0-mm layer of GIC (Maxxion R, FGM, Joinville, SC, Brazil) was used to cover the pulp chamber floor. The dentin was treated with $10 \%$ polyacrylic acid 
(Liquid of Maxxion R, FGM) for $10 \mathrm{~s}$, and was washed with water spray and carefully dried with absorbent paper. The GIC was inserted using a Centrix syringe (Maquira, Paraná, Brazil) to prevent syneresis and imbibition during the initial setting, and the occlusal surface of the tooth was covered with a pink wax blade for 5 min until the initial setting of the GIC. Selective etching of the enamel was performed using $37 \%$ phosphoric acid (Condac 37\%, FGM) for $30 \mathrm{~s}$, and a self-etching adhesive system (Clearfil SE Bond, Kuraray, Japan) was applied and light-cured for $20 \mathrm{~s}$. A conventional resin composite (Opalis, FGM) was inserted in 2.0-mm increments and light-cured for $40 \mathrm{~s}$ to complete the restoration. In group BFRC (Figure 1B), Opus Bulk Fill Flow (FGM) was used to fill the pulp chamber and replace the coronal dentin in 4-mm increments; the occlusal surface was restored using a conventional resin composite similar to that of group GIC. All materials were light-cured using a VALO Cordless (Ultradent Products, South Jordan, UT, USA) light-curing unit with an irradiance of $1400 \mathrm{~mW} / \mathrm{cm}^{2}$ checked using a MARC'M Resin Calibrator (BlueLight, Halifax, Canada).

\section{A}
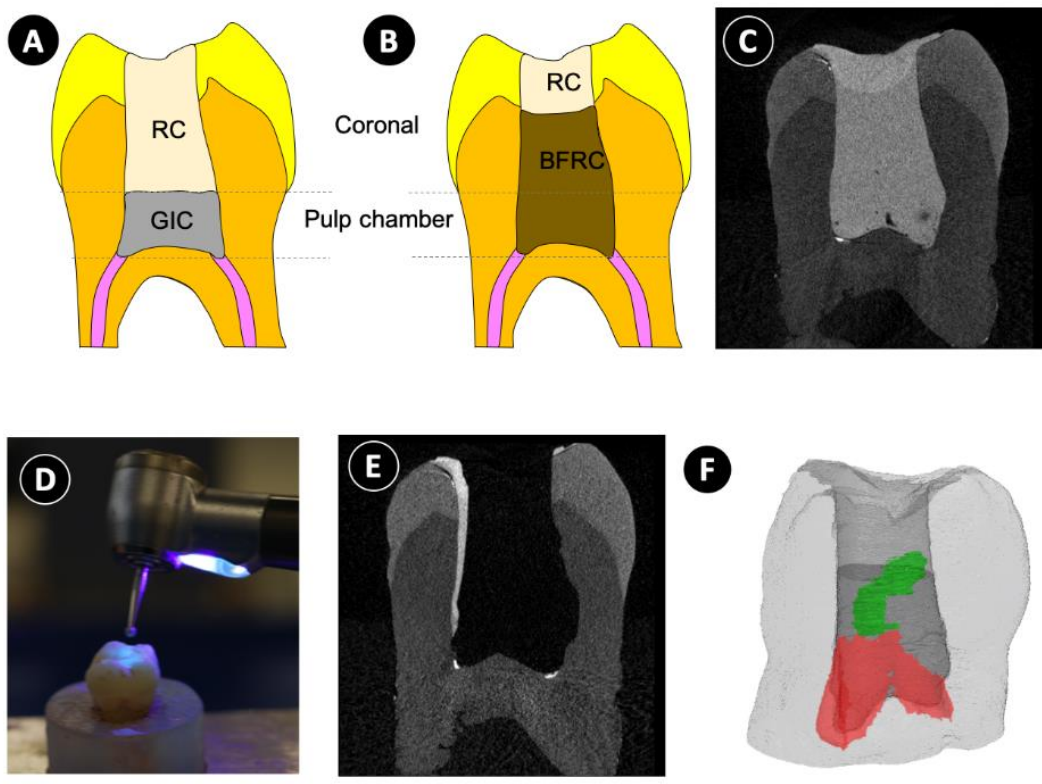

Figure 1. A. Schematic illustration of the glass ionomer cement with conventional resin composite group; B. Schematic illustration of the flowable bulk-fill resin composite with conventional composite resin group; $\mathrm{C}$. micro-CT image of the sample after endodontic treatment and coronal restoration; $D$. handpiece with ultraviolet light during re-access opening; E. micro-CT image of the sample after re-access opening; F. 3D volumetric superimposition of micro-CT before and after re-access opening; light gray-tooth structure; dark gray cavity preparation and restoration limit; green-dentin removal from the crown; red-dentin removal from the pulp chamber.

\section{Reaccess preparation in teeth}

The teeth in each restorative group were randomly sorted into two subgroups $(n=5)$ using www.random.org, and each subgroup was subjected to a different method to access the root canal for retreatment: Group WLHP, high-speed handpiece with white lighting (Kavo, Joinville, SC, Brazil) using 1.3-mm extra-long round diamond bur (\#1013 HL, KG Sorensen, Cotia, SP, Brazil); and Group VLHP, ultraviolet LED light high-speed handpiece (Ultra Vision Cobra LED, Gnatus, Ribeirão Preto, SP, Brazil) (Figure $1 \mathrm{C}$ ). Root canal access preparation was performed until the gutta-percha in the root canal orifices was identified.

\section{Micro-CT analysis}

To evaluate the amount of intact dentin removed during the access cavity preparation, the teeth were scanned after restoration and after re-access preparation using a micro-CT device (SkyScan 1272, Bruker, Belgium). The image acquisition took approximately $36 \mathrm{~min}$ for each tooth, with a standardized position, using the following parameters: an exposure time of $1800 \mathrm{~ms}$, energy of $100 \mathrm{KV}-100 \mu \mathrm{A}$, a $180^{\circ}$ rotation with a step of 0.700 , a $0.11-\mathrm{mm}$-thick $\mathrm{Cu}$ filter, and $15 \mu \mathrm{m}$ voxel size. 
The micro-CT images were imported to a computer and rebuilt using Nrecon software (version 1.6.10.1, SkyScan, Bruker, Belgium) with approximately 1.050 slices, respecting the anatomical limits of the samples. The restored tooth reference image (Figure 1D) and tooth image after endodontic re-access were superimposed (Figure 1E). The reconstructed images were overlaid using DataViewer software (version 1.5.1.2, SkyScan, Bruker, Belgium) and analyzed using the micro-CT analyzer software (CTAn, version 1.13, SkyScan, Bruker, Belgium) (14). A volume difference image (Diff), which represented the volume of dentin removed during root canal access for retreatment, was generated. Eight hundred layers, with a resolution of $0.4 \square \mathrm{m}$, were used to generate the volume of dentin removed in $\mathrm{mm}^{3}$, and the percentage was calculated as a function of the total volume of each tooth. Using CTVOL software (CTVol, version 2.0, SkyScan, Bruker, Belgium), 3D images of the volume of dentin removed were generated. The number and face (M: mesial, D: distal, B: buccal, and L: lingual) of the damaged dentin walls in the pulp chamber and crown (Figure 1A-1B) during the root canal access for endodontic retreatment were recorded (Figure 1F). The limit to determine the pulp chamber and crown area was the dentinoenamel junction.

\section{Statistical analysis}

The normality of the distributions (Shapiro Wilk's test) and the equality of variances (Levene's test) of the dentin volume removal $\left(\mathrm{mm}^{3}\right)$ data were tested, followed by parametric statistical tests. A twoway analysis of variance (ANOVA) was performed, followed by Tukey's test. The number and location of the affected dentin walls were described using frequency. All tests employed a significance level of 5\% $(\alpha=0.05)$ and were carried out with the statistical package Sigma Plot version 13.1 (Systat Software Inc., San Jose, CA, USA).

\section{Results}

The volume of dentin removed $\left(\mathrm{mm}^{3}\right)$ from the pulp chamber and from the inside of the coronal cavity during root canal re-access for endodontic retreatment are shown in Table 1. Two-way ANOVA revealed that the restorative material $(P<0.001)$ and handpiece light $(P<0.001)$ had significant effects on dentin removal from the pulp chamber. The material used $(P=0.812)$ and handpiece light $(P=0.452)$ did not influence the dentin removal from the coronal region. In general, the dentin removed from the pulp chamber was more than the coronal dentin removed. When GIC was used to fill the pulp chamber, dentin removal was significantly lesser than when flowable BFRC was used, irrespective of the handpiece light used (Figure 2). Critical dentin removal was visualized at the pulp chamber floor for groups restored with flowable BFRC (Figure 2).

Table 1. Mean and Standard deviation values of dentin volume wear $\left(\mathrm{mm}^{3}\right)$ during root canal access for endodontic retreatment.

\begin{tabular}{lcccc}
\hline \multirow{2}{*}{ Restorative Protocols } & \multicolumn{2}{c}{ White Light } & Ultraviolet Light \\
\cline { 2 - 5 } & $\begin{array}{c}\text { Pulp } \\
\text { Chamber }\end{array}$ & Crown & $\begin{array}{c}\text { Pulp } \\
\text { Chamber }\end{array}$ & Crown \\
\hline $\begin{array}{l}\text { Flowable bulk fill resin composite } \\
\text { /conventional resin composite }\end{array}$ & $8.8(1.1) \mathrm{Bb}$ & $3.3(1.6) \mathrm{Aa}$ & $11.4(3.2) \mathrm{Bb}^{*}$ & $4.9(1.2) \mathrm{Aa}$ \\
$\begin{array}{l}\text { Glass lonomer Cement/Incremental } \\
\text { Technique }\end{array}$ & $5.0(1.9) \mathrm{Aa}$ & $3.8(1.0) \mathrm{Aa}$ & $6.9(2.4) \mathrm{Ba}^{*}$ & $3.5(1.8) \mathrm{Aa}$
\end{tabular}

Different letters and ${ }^{*}$ represent significant differences between the factors. Capital letters used to compare tooth regions for each lighting mode; lower case letters used to compare restorative materials used to fill the pulp chamber. ${ }^{*}$ Used for comparing lighting modes within each region. Comparisons by Tukey's test $(\mathrm{P}<0.05)$. 

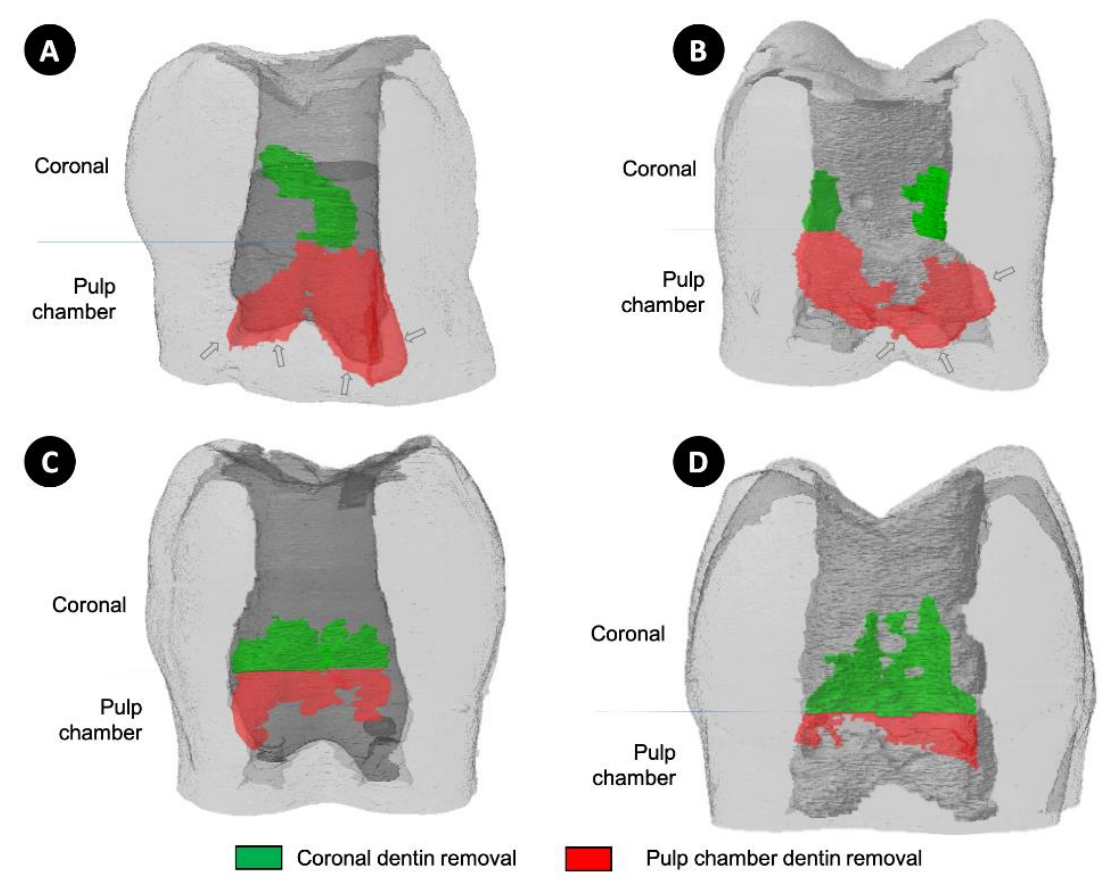

Figure 2. Volumetric micro-CT representation of dentin removed from the coronal and pulp chamber regions considering the restorative materials and handpiece light used for retreatment root canal access, superposing 3D images before and after re-access opening; light gray-tooth structure; dark gray cavity preparation and restoration limit; greendentin removal from the crown; red-dentin removal from the pulp chamber. $A$. handpiece with ultraviolet lighting/BFRC; $B$. handpiece with white lighting/BFRC; $C$. handpiece with ultraviolet lighting $/ G I C ; D$. handpiece with white lighting $/ \mathrm{GIC}$. Arrows illustrate the dentin removal in critical areas for groups $\mathrm{A}$ and $\mathrm{B}$.

The numbers and locations of the dentin walls affected during access for root canal retreatment are shown in Table 2 and Figure 2. The use of GIC to fill the pulp chamber reduced the number of dentin walls affected by dentin removal during access preparation. The buccal and lingual surfaces of the pulp chamber were the most affected areas in the pulp chamber during root canal access. The volumetric representation generated by micro-CT analysis is shown in Figure 2. Greater dentin removal was observed in the pulp chamber than in the coronal portion, mainly in the groups restored with flowable BFRC and reaccessed using a handpiece with violet light.

Table 2. Number and location of damaged dentin walls generated during re-access of root canals for endodontic retreatment.

\begin{tabular}{|c|c|c|c|c|c|c|c|c|c|c|c|c|c|c|c|c|}
\hline \multirow{3}{*}{$\begin{array}{l}\text { Restorative } \\
\text { Procedures }\end{array}$} & \multicolumn{9}{|c|}{ White Light } & \multicolumn{7}{|c|}{ Ultraviolet Light } \\
\hline & \multicolumn{4}{|c|}{ Pulp Chamber } & \multicolumn{4}{|c|}{ Crown } & \multicolumn{4}{|c|}{ Pulp Chamber } & \multicolumn{4}{|c|}{ Crown } \\
\hline & $M$ & D & B & $\mathrm{L}$ & $M$ & D & B & $\mathrm{L}$ & $M$ & $D$ & B & $\mathrm{L}$ & $M$ & D & B & $\mathrm{L}$ \\
\hline $\begin{array}{l}\text { Flowable bulk fill resin composite } \\
\text { /conventional resin composite }\end{array}$ & 2 & 3 & 3 & 2 & 2 & 0 & 1 & 1 & 1 & 0 & 5 & 3 & 2 & 1 & 3 & 1 \\
\hline $\begin{array}{l}\text { Glass lonomer Cement/Incremental } \\
\text { Technique }\end{array}$ & 1 & 1 & 4 & 5 & 1 & 2 & 1 & 2 & 0 & 3 & 3 & 1 & 1 & 2 & 2 & 1 \\
\hline
\end{tabular}

M: Mesial; D: Distal; B: Buccal; and L: Lingual. 


\section{Discussion}

The use of the white-light high-speed handpiece for re-access, along with the use of GIC to fill the pulp chamber reduced the volume of dentin removed from the pulp chamber and resulted in fewer affected dentin walls; therefore, the null hypothesis was rejected.

The methodological parameters and standardization of specimen preparation are fundamental for reducing in vitro study bias (17). The dimensions and shapes of the crowns and pulp chambers of the molar teeth were evaluated and were found to be similar in all groups. This parameter is important to prevent bias in dentin removal as root canal access preparation tend to be easier in larger teeth than in smaller teeth and variation occlusal anatomy may cause bias (18).

Although the success rate of endodontic treatment is close to 90\% (19), even well-performed root canals may fail (20). Endodontic retreatment is the most recommended option for managing these failures, and requires the removal of restorative materials for root canal access. Maintaining dentin integrity should be a goal when access to the root canal for retreatment is necessary, as intact dentin removal can increase tooth fracture after the final restoration (21). During root canal access for retreatment, a significant amount of dentin can be removed laterally and from the pulpal floor, which might lead to a higher occurrence of fractures (22).

Some factors may predispose to accidental dentin removal, which can weaken the tooth structure, and in the worst scenario, result in iatrogenic perforations (11). These events are found in clinical routine, whereas iatrogenic root dentin removal or perforations may occur during access cavity opening or root canal preparation (18). Testing the benefit of the lights present on the handpieces and the use of GIC inside the pulp chamber are clinically relevant for preventing these endodontic errors.

When retreatment is necessary, the restorative material used for filling cavities, mainly the pulp chamber of molar teeth, after endodontic treatment is a determinant of accidental dentin removal (23). The insertion of a flowable BFRC to fill the pulp chamber is easy, and reduces time and bubble formation. However, the similarity in color to that of root dentin (10) makes it difficult to identify the exact limit of the restoration during removal. The use of BFRC resulted in more intact dentin removal during root canal re-access preparation. Greater damage to the floor was verified, increasing the possibility of accidental perforation (7). In contrast, when the pulp chamber was filled using $\mathrm{GIC}$, lesser damage to the pulpal floor was observed. The color similarity and the higher stiffness of BFRC require more pressure during removal, increasing the possibility of undesirable contact between the bur and the root dentin. Removal of GIC from the floor of the pulp chamber was facilitated by better visualization, and the texture and lower stiffness of the material (8). In some cases, the use of only an exploratory probe with a straight and sharp tip was sufficient to remove the GIC from the walls, resulting in less accidental dentin removal. This study demonstrated that using GIC to fill the pulp chamber and seal the entrance to the root canal in molar teeth is recommended for reducing dentin removal during access preparation for root canal retreatment.

An incomplete access cavity reduces the quality of emptying and may alter the ideal shape of the root canal preparation. An exaggerated access cavity favors root perforation and makes the tooth susceptible to coronal/radicular fractures (11). Residual resin composite can create an obstacle for endodontic file access, making correct instrumentation difficult. To avoid this situation, a straight-line access to the canals is necessary to enhance instrumentation efficacy and prevent procedural errors (24). The 3D reconstruction of the molar teeth after root canal access for retreatment showed a greater presence of residual resin composite on the lateral coronal dentin in the pulp chamber, which contributed negatively to future root canal instrumentation.

The use of ultraviolet light was tested to confirm whether the fluorescence of the resin composite could facilitate the identification of the dentin/restorative material limit. Ultraviolet light helped to identify the material only at the occlusal surface during the initial removal because the light intensity was able to highlight the fluorescence of the resin composite $(13,14)$. This property is used for fluorescence-aided composite removal during lingual bracket debonding, as it makes resin composite removal easier, more accurate, reliable, noninvasive, and time-saving (12). Two recent studies demonstrated the effectiveness of the fluorescence-aided identification technique to preserve tooth structure during resin composite restoration removal $(25,26)$. However, ultraviolet light was ineffective in the pulp chamber. This can be explained by the limited penetration depth of ultraviolet light and the need to be used at a lower intensity due to potential health hazards (27). The manufacturer of the handpiece Ultra Vision, tested in this study, recommends the use of goggles for safety, which confirms 
its additional disadvantage compared to the handpiece with white light. The 25,000 Lux generated by the daylight-neutral 5,500 K color temperature illumination presented in the LED KAVO handpiece allows proper penetration inside the root canal preparation, contributing to better visualization of the dentin/restorative material limit. Another explanation for the different results could be related to the wavelength and power of the light added to the handpiece. The use of a 405-nm-wavelength light with higher power can improve the quality of resin visualization $(25,26)$. The fluorescence-aided identification tends to depend on the handpiece quality.

This study has some limitations: the sample selection was performed using digital radiographs, and standardization of the shape and volume could be a concern during the experimental design, as these data can be determined by the parameters obtained by micro-CT (28). Only one conventional GIC and one BFRC were included in this study. Different glass ionomers mainly resin-modified GIC that has a higher color similarity to the dentin can perform differently during removal from deep areas. Additionally, we tested only one shade of the resin composite; using a clear shade, such as A1 or B1, in the pulp chamber can possibly facilitate the differentiation from the dentin. Another limitation of this study was the absence of a test group with a handpiece without light as a control. These aspects should be considered in future studies, which should also analyze the effect of dentin removal on the biomechanical performance of restored and non-restored teeth. The sample size was limited, but it was sufficient to demonstrate a significant difference in the effect of the study factors on dentin removal in the pulp chamber.

In conclusion, the use of GIC to fill the pulp chamber reduces accidental dentin removal. The use of handpieces with ultraviolet light offers no advantage in preventing dentin removal. The benefit of using a handpiece with light observed in this study can also be extrapolated to the first root canal access, contributing to more conservative endodontic procedures. When using a handpiece with white light, the removal of intact dentin was lesser than when using a handpiece with ultraviolet light, irrespective of the restorative material used. Extra caution should be exercised when removing restorative material located on the palatal and especially on the buccal walls of the pulp chamber.

\section{Acknowledgement}

This project was funded by grants from CNPq-National Council for Scientific and Technological Development, grants 434598/2018-6; and FAPEMIG, grants APQ-02105-18.

\section{Resumo}

Este estudo avaliou os efeitos do cimento de ionômero de vidro (GIC) e da resina composta fluida bulk fill (BFRC) usados como preenchimento da câmara pulpar; e o tipo de iluminação das turbinas de alta rotação na remoção dentinária após cavidades de acesso para retratamento endodôntico em dentes molares. Vinte molares superiores foram tratados endodonticamente. Dez dentes foram restaurados usando BFRC (Opus Bulk Fill Flow APS, FGM) para preencher a câmara pulpar e dentina coronária; e resina composta convencional (Opallis, FGM) para restaurar a camada de esmalte. Os outros dentes foram restaurados usando GIC (Maxion R, FGM) para preencher a câmara pulpar e resina composta (Opallis, FGM). As amostras foram divididas em dois grupos e os canais radiculares foram novamente acessados com turbina de alta-rotação com iluminação branca ou ultravioleta. Os dentes foram escaneados usando micro-CT antes e após o novo acesso ao canal radicular. 0 volume de dentina removida foi calculado e os dados foram analisados por ANOVA bidirecional e teste de Tukey $(\alpha=0,05)$. As regiões na coroa e na câmara pulpar que apresentaram dentina removida no acesso dos canais foram descritas por meio de distribuição por frequência. A reabertura do canal radicular com GIC resultou em menos paredes afetadas da câmara pulpar e menor volume de dentina removida no assoalho. Nenhum efeito foi observado nas paredes de dentina coronária considerando aos protocolos de preenchimento. A turbina de alta rotação com iluminação branca reduziu a remoção de dentina da câmara pulpar, independentemente do protocolo de restauração utilizado. 0 uso de turbina de alta rotação com iluminação branca e GIC para preencher a câmara pulpar reduziram a remoção de dentina do assoalho e afetaram menos paredes dentinárias. 


\section{References}

1. Milani AS, Froughreyhani M, Mohammadi $\mathrm{H}$, Tabegh FG, Pournaghiazar F. The effect of temporary restorative materials on fracture resistance of endodontically treated teeth. Gen Dent 2016;64:e1-4.

2. Pereira R, Bicalho AA, Franco SD, Tantbirojn D, Versluis A, Soares CJ. Effect of Restorative Protocol on Cuspal Strain and Residual Stress in Endodontically Treated Molars. Oper Dent 2016;41:23-33.

3. Soares CJ, Rodrigues MP, Faria ESAL, Santos-Filho PCF, Verissimo C, Kim HC, et al. How biomechanics can affect the endodontic treated teeth and their restorative procedures? Braz Oral Res 2018;32:e76.

4. Wiegand A, Kanzow P. Effect of Repairing Endodontic Access Cavities on Survival of Single Crowns and Retainer Restorations. J Endod 2020;46:376-382.

5. Tani K, Watari F, Uo M, Morita M. Discrimination between composite resin and teeth using fluorescence properties. Dent Mater J 2003;22:569-580.

6. Alrahabi M, Zafar MS, Adanir N. Aspects of Clinical Malpractice in Endodontics. Eur J Dent 2019;13: 450-458.

7. Silveira CM, Sanchez-Ayala A, Lagravere MO, Pilatti GL, Gomes OM. Repair of furcal perforation with mineral trioxide aggregate: long-term follow-up of 2 cases. J Can Dent Assoc 2008;74:729-733.

8. Kheur M, Kantharia N, Lakha T, Kheur S, Al-Haj Husain N, Ozcan M. Evaluation of mechanical and adhesion properties of glass ionomer cement incorporating nano-sized hydroxyapatite particles. Odontology 2020;108:66-73.

9. Lachowski KM, Botta SB, Lascala CA, Matos AB, Sobral MA. Study of the radio-opacity of base and liner dental materials using a digital radiography system. Dentomaxillofac Radiol 2013;42: 20120153.

10. Bucuta $S$, Ilie N. Light transmittance and micro-mechanical properties of bulk fill vs. conventional resin based composites. Clin Oral Investig 2014;18: 1991-2000.

11. Estrela C, Pécora JD, Estrela CRA, Guedes OA, Silva BS, Soares CJ et al. Common operative procedural errors and clinical factors associated with root canal treatment. Braz Dent J. 2017;28:179190.

12. Albertini $P$, Albertini $E$, Siciliani G, Lombardo L. Fluorescence-aided composite removal during lingual bracket debonding. J Esthet Restor Dent. 2020;32(7):634-637.

13. Guzy G, Clayton MA. Detection of composite resin restorations using an ultraviolet lightemitting diode flashlight during forensic dental identification. Am J Forensic Med Pathol 2013;34:8689.

14. Salomao FM, Rocha RS, Franco LM, Sundfeld RH, Bresciani E, Fagundes TC. Auxiliary UV light devices for removal of fluorescent resin residues after bracket debonding. J Esthet Restor Dent 2019;31:58-63.

15. Oliveira LRS, Braga SSL, Bicalho AA, Ribeiro MTH, Price RB, Soares CJ. Molar cusp deformation evaluated by micro-CT and enamel crack formation to compare incremental and bulk-filling techniques. J Dent 2018;74:71-78.

16. Soares CJ, Pizi EC, Fonseca RB, Martins LR. Influence of root embedment material and periodontal ligament simulation on fracture resistance tests. Braz Oral Res 2005;19:11-16.

17. Krithikadatta J, Gopikrishna V, Datta M. CRIS Guidelines (Checklist for Reporting In-vitro Studies): A concept note on the need for standardized guidelines for improving quality and transparency in reporting in-vitro studies in experimental dental research. J Conserv Dent. 2014;17(4):301-304.

18. Estrela C, Decurcio DA, Rossi-Fedele G, Silva JA, Guedes OA, Borges ÁH. Root perforations: a review of diagnosis, prognosis and materials. Braz Oral Res. 2018;18:32(suppl 1)-e73.

19. Lewis RD, Block RM. Management of endodontic failures. Oral Surg Oral Med Oral Pathol 1988;66:711-721.

20. Siqueira JF, Jr. Aetiology of root canal treatment failure: why well-treated teeth can fail. Int Endod J 2001;34:1-10.

21. Krishan $R$, Paque $F$, Ossareh A, Kishen A, Dao T, Friedman S. Impacts of conservative endodontic cavity on root canal instrumentation efficacy and resistance to fracture assessed in incisors, premolars, and molars. J Endod 2014;40:1160-1166.

22. Zhi-Yue L, Yu-Xing Z. Effects of post-core design and ferrule on fracture resistance of endodontically treated maxillary central incisors. J Prosthet Dent 2003;89:368-373.

23. Agrafioti A, Giannakoulas DG, Kournetas N, Grigoriou S, Kontakiotis EG. Different Patterns of Restoration Provision Between Initial Endodontic Treatment and Retreatment: A Retrospective Clinical Study. Int J Prosthodont 2017;30:354-356. 
24. Schroeder KP, Walton RE, Rivera EM. Straight line access and coronal flaring: effect on canal length. J Endod 2002;28:474-476.

25. Klein C, Babai A, von Ohle $C$, Herz M, Wolff D, Meller $C$. Minimally invasive removal of toothcolored restorations: evaluation of a novel handpiece using the fluorescence-aided identification technique (FIT). Clin Oral Investig. 2020;24(8):2735-2743.

26. Dettwiler C, Eggmann F, Matthisson L, Meller C, Weiger R, Connert T. Fluorescence-aided Composite Removal in Directly Restored Permanent Posterior Teeth. Oper Dent. 2020;45(1):62-70.

27. Santini A, Gallegos IT, Felix CM. Photoinitiators in dentistry: a review. Prim Dent J. 2013;2(4):3033.

28. Sousa-Neto MD, Silva-Sousa YC, Mazzi-Chaves JF, Carvalho KKT, Barbosa AFS, Versiani MA, Jacobs R, Leoni GB. Root canal preparation using micro-computed tomography analysis: a literature review. Braz Oral Res. 2018;32(suppl 1):e66.

Received: 25/08/2021

Accepted: 24/09/2021 